\title{
A population-based analysis of chemoradiation versus radiation alone in the definitive treatment of patients with stage I-II squamous cell carcinoma of the anus
}

\author{
Jacob S. Parzen, Aleksander Vayntraub, Bryan Squires, Muayad F. Almahariq, Andrew B. Thompson, \\ John M. Robertson, Peyman Kabolizadeh, Thomas J. Quinn
}

Beaumont Health, Department of Radiation Oncology, Royal Oak, MI, USA

Contributions: (I) Conception and design: TJ Quinn; (II) Administrative support: TJ Quinn; (III) Provision of study materials or patients: TJ Quinn; (IV) Collection and assembly of data: TJ Quinn; (V) Data analysis and interpretation: JS Parzen, P Kabolizadeh, TJ Quinn; (VI) Manuscript writing: All authors; (VII) Final approval of manuscript: All authors.

Correspondence to: Thomas J. Quinn, MD. Department of Radiation Oncology, 18101 Oakwood Boulevard, BH-D Radiation Oncology Department, Lower Level, Dearborn, MI 48124, USA. Email: Thomas.Quinn@beaumont.org.

Background: The optimal management of patients with stage I-II squamous cell carcinoma (SCC) of the anus is controversial. The current study evaluates the efficacy of combined chemotherapy and radiation therapy (CRT) versus radiation therapy (RT) alone in the treatment of these patients using the Surveillance, Epidemiology, and End Results (SEER) registries.

Methods: SEER 18 Custom Data registries were queried for patients with stage I-II SCC of the anus. Univariate analysis (UVA) and multivariable analysis (MVA) using Kaplan-Meier and Cox proportional hazards regression modeling were performed. Propensity-score matched analysis with inverse probability of treatment weighting (IPTW) was used to account for indication bias.

Results: A total of 4,288 patients with stage I-II disease were identified, of whom 3,982 (93\%) underwent CRT and 306 (7\%) underwent RT. Median follow-up was 42 months. Approximately 30.8\% had T1 disease and $69.2 \%$ had T2-T3 disease. The IPTW-adjusted 5 -year overall survival (OS) was 76.7\%, with no significant differences between the CRT and RT groups ( $77 \%$ s. $73.5 \%, \mathrm{P}=0.33)$. On multivariate IPTWadjusted analysis, the lack of association between CRT use and OS was upheld (HR, 0.84, 95\% CI, 0.65-1.08, $\mathrm{P}=0.2)$. On subgroup analyses, 5 -year OS was $86 \%$ with CRT $(\mathrm{n}=1,216)$ and $84.2 \%$ with $\mathrm{RT}(\mathrm{n}=103)(\mathrm{P}=0.74)$ in stage I (T1N0) patients, while 5-year OS was 72.8\% with CRT $(\mathrm{n}=2,766)$ and $66.4 \%$ with RT $(\mathrm{n}=203)$ $(\mathrm{P}=0.13)$ in stage II (T2-3N0) patients. CRT was associated with improved median OS in stage II patients (119 months $v s$. not reached, $\mathrm{P}=0.04)$.

Conclusions: The current study suggests that omission of concurrent chemotherapy is not associated with inferior OS in patients with stage I SCC of the anus. However, combined chemoradiation was superior to radiation alone in patients with stage II disease. Prospective evidence is needed to optimize clinical decisionmaking in this patient population.

Keywords: Squamous cell carcinoma of anus; chemoradiation; radiation monotherapy; population-based study

Submitted Nov 20, 2020. Accepted for publication Jan 17, 2021.

doi: 10.21037/jgo-20-530

View this article at: http://dx.doi.org/10.21037/jgo-20-530 


\section{Introduction}

The incidence of anus cancer is rising in the United States, with an estimated 8,590 cases and 1,350 deaths attributed to the disease in 2020 (1). Importantly, the proportion of earlystage and in situ disease has also increased (2). Historically, patients with anus cancer were all treated surgically, rendering them colostomy-dependent (3). In the 1970s, the Nigro regimen accelerated the movement towards organ preservation, with an $86 \%$ complete clinical response rate with chemoradiation (CRT) (4). Two large randomized trials from the European Organization for Research and Treatment of Cancer (EORTC) and United Kingdom Coordinating Committee on Cancer Research (UKCCCR) subsequently established concurrent CRT as the standard of care over radiation therapy (RT) alone in patients with anal cancer $(5,6)$. However, the optimal management of patients with T1-2N0 disease remains controversial due to underrepresentation on these studies. The UKCCCR group did report a local control benefit in the 223 (38\%) patients with T1N0 and T2N0 disease, though this was an unplanned subset analysis (7). The benefit of chemotherapy was corroborated on several other studies (8-11).

In contrast, a number of historical series and more recent population-based studies suggest that omission of concurrent chemotherapy has no deleterious effect in patients with T1 lesions (12-16). Nonetheless, the National Comprehensive Cancer Network (NCCN) guidelines recommend concurrent CRT and do not offer RT monotherapy as an alternative in patients with T1T2N0 disease (17). This is despite the fact that the most common concurrent chemotherapy regimen, fluorouracil and mitomycin $\mathrm{C}$, results in significant, sometimes lifethreatening, hematological toxicity (18).

The present study uses population data from the Surveillance, Epidemiology, and End Results (SEER) registries to evaluate the comparative efficacy of concurrent CRT and RT as monotherapy in patients with stage III squamous cell carcinoma (SCC) of the anus. We hypothesized that patients with T1 lesions $(\leq 2 \mathrm{~cm})$ would not benefit appreciably from the addition of concurrent chemotherapy. We present the following article in accordance with the STROBE reporting checklist (available at: http://dx.doi.org/10.21037/jgo-20-530).

\section{Methods}

This study was exempt from institutional review board approval because the data set was de-identified and publicly available. The study was conducted in accordance with the Declaration of Helsinki (as revised in 2013).

\section{Data source}

The SEER Program publishes cancer incidence and survival data for approximately $34 \%$ of the US population. The specialized Radiation/Chemotherapy Database (SEER 18 Custom Data, November 2018 Submission) was used as it contains information on RT and chemotherapy. Of note, the chemotherapy field is coded either as yes or none/ unknown.

\section{Cohort analyzed}

We queried the SEER 18 Custom Data registries for International Classification of Disease (ICD-10) C21.0-C21.8, with histologic confirmation of malignancy, corresponding to malignant neoplasms of the anus. Patients with ICD-10 C44.520, corresponding to squamous cell cancer of the anal skin, were not included. Patients were excluded as detailed in the CONSORT diagram (Figure 1). From 2004 to 2016, a total of 4,288 patients with stage III SCC of the anus who received external beam RT were identified. Among this cohort, 3,982 received CRT and 306 received RT alone. We verified that stage I disease corresponded to T1 lesions and that stage II disease corresponded to T2-3 lesions. We also verified that tumor size corresponded to appropriate $\mathrm{T}$ staging. This study was exempt from institutional review board approval because the data set was de-identified and publicly available.

\section{Statistical analysis}

Baseline patient characteristics were assessed before and after matching with chi-squared analysis and standard mean difference (SMD), where a $\mathrm{SMD}>0.1$ was considered unbalanced (19). Univariate analysis (UVA) of the impact of patient characteristics on overall survival (OS) was performed using the Kaplan-Meier (KM) method, with the $\log$ rank method to assess for significance (20). Multivariable analysis (MVA) of patient characteristics and OS was performed using Cox proportional hazards regression modeling. Covariates with $\mathrm{P}<0.1$ in the UVA were incorporated in multivariable cox proportional hazards regression modeling using backward stepwise methodology to mitigate collinearity of variables and overfitting of the 


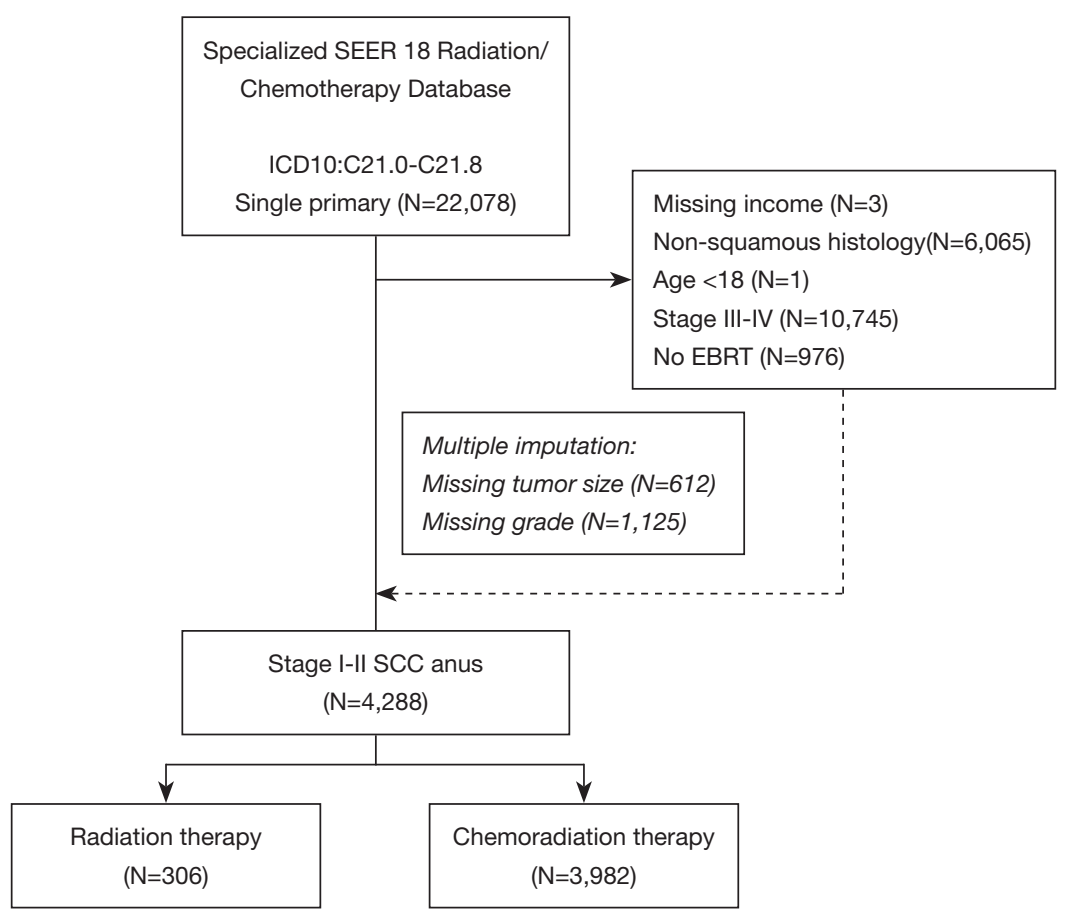

Figure 1 CONSORT diagram demonstrating the inclusion and exclusion criteria used to select stage I-II squamous cell carcinoma (SCC) of the anus.

final MVA model (21).

Multiple imputation by chained equations was used to replace missing data $(22,23)$. In particular, multiple imputations were performed for the SEER parameters Tumor Size Summary 2016 and Grade. We performed a propensity score-matched analysis to account for indication bias. Propensity scores (PS) were estimated using binary logistic regression modeling for receipt of CRT or RT (24). Inverse probability of treatment weights (IPTW) were then calculated as 1/PS and 1/(1-PS) (25). We performed stabilization of the IPTWs by multiplying the standard IPTWs by the probability of undergoing treatment that each patient received (26). Finally, we performed IPTWadjusted UVA and doubly robust, IPTW-adjusted MVA as detailed previously $(27,28)$.

We completed all statistics using SEER*Stat (v8.3.5, The Surveillance Research Program of the Division of Cancer Control and Population Sciences, National Cancer Institute) and $\mathrm{R}$ version 3.6.2 statistical software ( $\mathrm{R}$ Foundation for Statistical Computing, Vienna, Austria). All statistical analyses were performed as two-sided with $\mathrm{P}<0.05$ considered statistically significant. $\mathrm{R}$ markdown for all analyses is available upon request.

\section{Results}

\section{Patient and tumor characteristics}

We identified 4,288 patients with stage I-II SCC of the anus in the SEER registries, of whom 3,982 (92.9\%) underwent CRT and 306 (7.1\%) underwent RT alone. Table 1 depicts patient and tumor characteristics for the unadjusted populations and the propensity score-matched patients. Overall median follow-up was 42 months, similar between CRT and RT groups. Patients in the RT group were more likely to be African-American $(\mathrm{P}=0.01)$, less likely to be insured $(\mathrm{P}=0.01)$, more likely to be widowed $(\mathrm{P}<0.001)$, and more likely to be diagnosed at an earlier year $(\mathrm{P}<0.001)$. Following propensity score matching with IPTW, there were no significant differences in baseline characteristics between the CRT and RT groups.

\section{UVA}

The impact of patient, tumor, and treatment characteristics on OS were evaluated using the Kaplan-Meier method. Table 2 provides the UVA for the unadjusted and propensity-matched IPTW-adjusted CRT and RT groups. 
Table 1 Patient and tumor characteristics

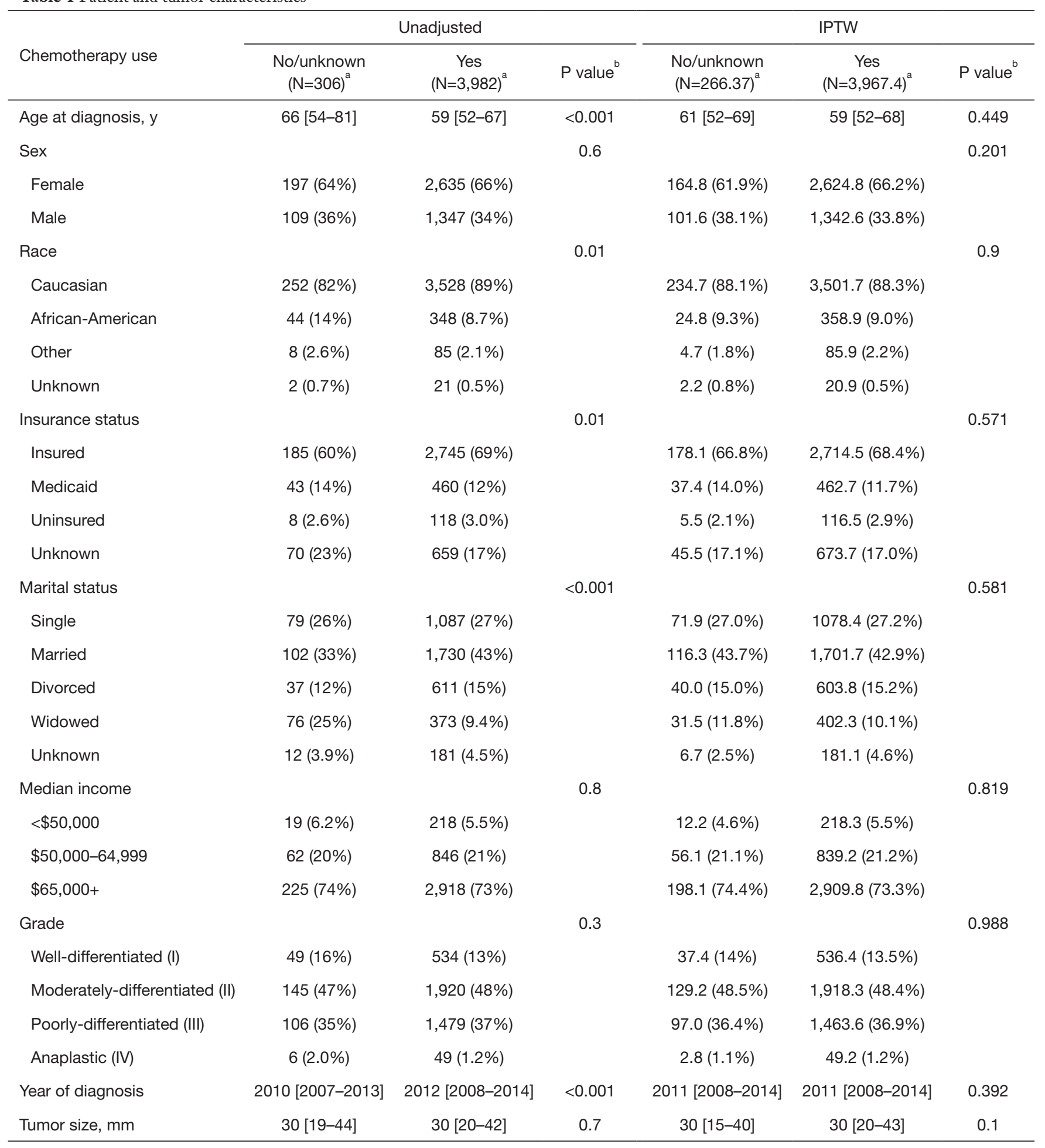

Table 1 (continued) 
Table 1 (continued)

\begin{tabular}{|c|c|c|c|c|c|c|}
\hline Chemotherapy use & \multicolumn{3}{|c|}{ Unadjusted } & \multicolumn{3}{|c|}{ IPTW } \\
\hline T stage & & & 0.1 & & & 0.478 \\
\hline $\mathrm{T} 1$ & $103(34 \%)$ & 1,215 (31\%) & & 93.7 (35.2\%) & 1,211.8 (30.5\%) & \\
\hline Unknown & $0(0 \%)$ & $3(<0.1 \%)$ & & $0.0(0.0 \%)$ & $2.9(<0.1 \%)$ & \\
\hline Overall stage & & & 0.3 & & & 0.161 \\
\hline I & 103 (34\%) & $1,216(31 \%)$ & & 93.7 (35.2\%) & 1,212.8 (30.6\%) & \\
\hline
\end{tabular}

${ }^{a}$, Statistics presented: median (interquartile range); $\mathrm{n}(\%){ }^{\mathrm{b}}$, Statistical tests performed: Wilcoxon rank-sum test; chi-square test of independence; Fisher's exact test. IPTW, inverse probability of treatment weighting; m, months; y, years; mm, millimeters.

In the unadjusted population, older age at diagnosis, male sex, African-American race, Medicaid recipient status, widowed status, anaplastic histology, tumor size groupings $\geq 2.6 \mathrm{~cm}, \mathrm{~T} 2 / \mathrm{T} 3$ status, and stage II disease were poor prognostic features. Conversely, married status, median income $\geq \$ 65,000$, later year at diagnosis and receipt of chemotherapy (HR 0.47, 95\% CI, 0.39-0.57, P<0.001) were protective. However, after propensity score matching and IPTW, the use of chemotherapy was no longer associated with improved OS for the entire cohort analyzed (HR 0.85, $95 \%$ CI, 0.67-1.08, $\mathrm{P}=0.2$ ).

\section{MVA}

Overall stage and $\mathrm{T}$ stage were eliminated from the MVA due to co-linearity with tumor size categories. Table 3 provides the MVA for the unadjusted and propensitymatched IPTW-adjusted CRT and RT groups. For the unadjusted groups, older age at diagnosis, male sex, AfricanAmerican race, Medicaid recipient status, widowed status, anaplastic histology, and tumor size groupings $\geq 2.6 \mathrm{~cm}$, were negative prognostic factors. Married status, median income $\geq \$ 65,000$, later year at diagnosis, tumor size group $1-1.5 \mathrm{~cm}$, and receipt of chemotherapy (HR $0.66,95 \% \mathrm{CI}$, $0.54-0.80, \mathrm{P}<0.001)$ were associated with improved survival. After propensity score matching with IPTW, the use of chemotherapy was no longer associated with improved OS (HR 0.84, 95\% CI, 0.65-1.08, $\mathrm{P}=0.2$ ).

\section{OS by treatment}

The 5-year OS for the entire cohort was $76.1 \%$ (95\% CI, 74.6-77.6\%) before and 76.7\% (95\% CI, 75.2-78.3\%) after propensity score matching with IPTW. Prior to matching, the RT group had a lower 5 -year OS $(57.9 \%, 95 \%$ CI, $52-$ $64.5 \%)$ than the CRT group (77.7\%, 95\% CI, 76.1-79.2\%, $\mathrm{P}<0.001$ ) (Figure $2 A$ ). After propensity score matching with IPTW, there was no difference in 5 -year OS between the RT group (73.5\%, 95\% CI, 67.7-79.9\%) and the CRT group (77\%, 95\% CI, 75.4-78.6\%, $\mathrm{P}=0.33$ ) (Figure $2 B$ ). The unadjusted and IPTW-adjusted 10-year OS rates were $66.9 \%$ and $66 \%$ for the CRT group and $41.2 \%$ and $56.5 \%$ for the RT group, respectively. There was no statistically significant difference in 10-year OS following propensity score matching and IPTW $(\mathrm{P}=0.23)$.

\section{OS for stage I and II SCC of the anus}

The 5-year OS for the subset of stage I patients was $85.2 \%$ (95\% CI, 83-87.5\%) before and $85.9 \%$ (95\% CI, $83.7-$ $88.2 \%)$ after propensity score matching with IPTW. Prior to matching, the RT group had a lower 5-year OS $(70.1 \%$, 95\% CI, 60.3-81.4\%) than the CRT group (86.6\%, 95\% CI, 84.4-88.9\%, $\mathrm{P}<0.001$ ) (Figure 3A). After propensity score matching with IPTW, there was no difference in 5 -year OS between the RT group (84.2\%, 95\% CI, 76.8$92.4 \%)$ and the CRT group (86\%, 95\% CI, 83.7-88.4\%, 
Table 2 Univariate Cox proportional hazards analysis

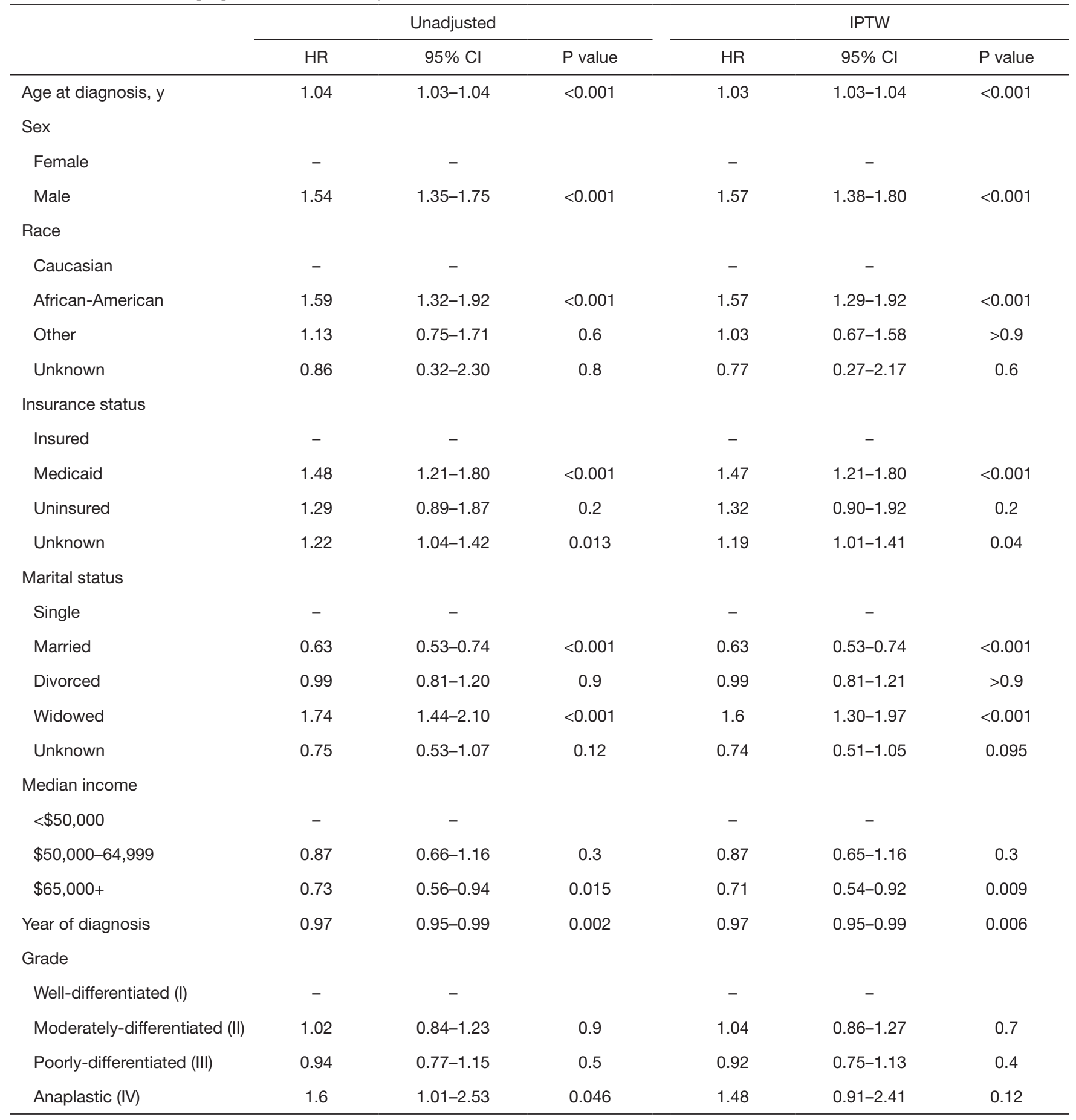

Table 2 (continued) 
Table 2 (continued)

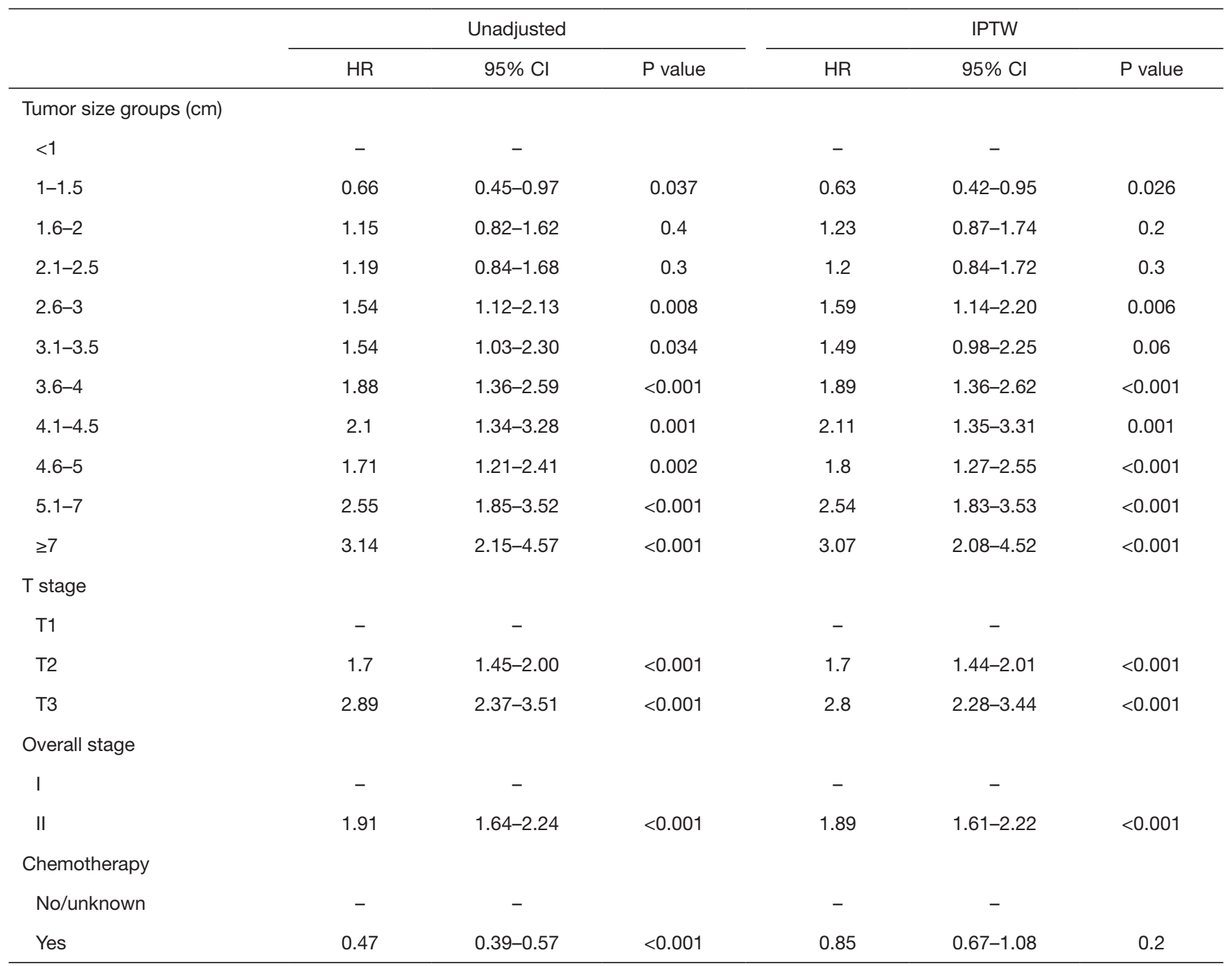

$\mathrm{Cl}$, confidence interval; HR, hazard ratio; y, years; $\mathrm{cm}$, centimeters.

$\mathrm{P}=0.74$ ) (Figure 3B). The unadjusted and IPTW-adjusted 10 -year OS rates were $75.2 \%$ and $74.1 \%$ for the CRT group and $59 \%$ and $73.2 \%$ for the RT group, respectively. There was no statistically significant difference in 10-year OS following propensity score matching and IPTW $(\mathrm{P}=0.98)$.

The 5 -year OS for the subset of stage II patients was $72 \%$ (95\% CI, 70.1-73.9\%) before and $72.4 \%$ (95\% CI, 70.4-74.4\%) after propensity score matching with IPTW. Prior to matching, the RT group had a lower 5-year OS $(51.6 \%, 95 \% \mathrm{CI}, 44.5-59.9 \%)$ than the CRT group (73.6\%, 95\% CI, 71.7-75.6\%, $\mathrm{P}<0.001$ ) (Figure 3C). After propensity score matching with IPTW, there was no difference in 5 -year OS between the RT group (66.4\%, $95 \%$ CI, $58.5-75.2 \%)$ and the CRT group $(72.8 \%, 95 \%$ CI, $70.7-74.8 \%, \mathrm{P}=0.13$ ); however, there was a statistically significant improvement in the median OS favoring the CRT group (119 months vs. NR, $\mathrm{P}=0.04$ ) (Figure 3D). Subset analyses were completed comparing CRT and RT for tumor sizes $<1,1-2,2.1-3,3.1-5,5.1-7$, and $>7 \mathrm{~cm}$ (Figure S1).

\section{Discussion}

This large population-based cohort analysis fails to reveal a difference in OS between CRT and RT alone in patients 
Table 3 Multivariable Cox proportional hazards analysis

\begin{tabular}{|c|c|c|c|c|c|c|}
\hline & \multicolumn{3}{|c|}{ Unadjusted } & \multicolumn{3}{|c|}{ IPTW } \\
\hline Age at diagnosis, y & 1.04 & $1.03-1.04$ & $<0.001$ & 1.04 & $1.03-1.04$ & $<0.001$ \\
\hline \multicolumn{7}{|l|}{ Sex } \\
\hline Female & - & - & & - & - & \\
\hline \multicolumn{7}{|l|}{ Race } \\
\hline Caucasian & - & - & & - & - & \\
\hline African-American & 1.54 & $1.26-1.88$ & $<0.001$ & 1.53 & $1.25-1.87$ & $<0.001$ \\
\hline Other & 1.01 & $0.66-1.53$ & $>0.9$ & 0.94 & $0.60-1.47$ & 0.8 \\
\hline \multicolumn{7}{|l|}{ Insurance status } \\
\hline Insured & - & - & & - & - & \\
\hline Medicaid & 1.34 & $1.09-1.64$ & 0.006 & 1.28 & $1.04-1.57$ & 0.02 \\
\hline Uninsured & 1.42 & $0.97-2.09$ & 0.074 & 1.41 & $0.95-2.09$ & 0.085 \\
\hline Unknown & 1.04 & $0.84-1.30$ & 0.7 & 1.02 & $0.82-1.28$ & 0.9 \\
\hline \multicolumn{7}{|l|}{ Marital status } \\
\hline Single & - & - & & - & - & \\
\hline Married & 0.75 & $0.63-0.89$ & 0.001 & 0.73 & $0.61-0.88$ & $<0.001$ \\
\hline Divorced & 1.09 & $0.89-1.34$ & 0.4 & 1.09 & $0.88-1.34$ & 0.4 \\
\hline$\$ 65,000+$ & 0.77 & $0.59-0.99$ & 0.046 & 0.73 & $0.56-0.95$ & 0.021 \\
\hline Year of diagnosis & 0.96 & $0.93-0.99$ & 0.007 & 0.95 & $0.92-0.98$ & 0.003 \\
\hline \multicolumn{7}{|l|}{ Grade } \\
\hline Well-differentiated (I) & - & - & & - & - & \\
\hline Moderately-differentiated (II) & 1.14 & $0.94-1.40$ & 0.2 & 1.17 & $0.96-1.44$ & 0.12 \\
\hline Poorly-differentiated (III) & 1.09 & $0.88-1.34$ & 0.4 & 1.07 & $0.86-1.32$ & 0.5 \\
\hline Anaplastic (IV) & 2.37 & $1.48-3.78$ & $<0.001$ & 2.23 & $1.36-3.67$ & 0.002 \\
\hline
\end{tabular}

Table 3 (continued) 
Table 3 (continued)

\begin{tabular}{|c|c|c|c|c|c|c|}
\hline & \multicolumn{3}{|c|}{ Unadjusted } & \multicolumn{3}{|c|}{ IPTW } \\
\hline \multicolumn{7}{|c|}{ Tumor size groups $(\mathrm{cm})$} \\
\hline$<1$ & - & - & & - & - & \\
\hline $1-1.5$ & 0.65 & $0.44-0.96$ & 0.03 & 0.64 & $0.43-0.95$ & 0.029 \\
\hline $2.1-2.5$ & 1.07 & $0.76-1.52$ & 0.7 & 1.1 & $0.77-1.57$ & 0.6 \\
\hline $2.6-3$ & 1.46 & $1.06-2.02$ & 0.021 & 1.47 & $1.06-2.04$ & 0.022 \\
\hline $3.1-3.5$ & 1.6 & $1.07-2.39$ & 0.023 & 1.51 & $0.99-2.28$ & 0.054 \\
\hline $3.6-4$ & 1.72 & $1.24-2.38$ & 0.001 & 1.73 & $1.24-2.41$ & 0.001 \\
\hline $5.1-7$ & 2.25 & $1.62-3.11$ & $<0.001$ & 2.21 & $1.59-3.08$ & $<0.001$ \\
\hline$\geq 7$ & 2.82 & $1.93-4.12$ & $<0.001$ & 2.81 & $1.90-4.16$ & $<0.001$ \\
\hline \multicolumn{7}{|l|}{ Chemotherapy } \\
\hline No/unknown & - & - & & - & - & \\
\hline Yes & 0.66 & $0.54-0.80$ & $<0.001$ & 0.84 & $0.65-1.08$ & 0.2 \\
\hline
\end{tabular}

$\mathrm{Cl}$, confidence interval; $\mathrm{HR}$, hazard ratio; y, years; $\mathrm{cm}$, centimeters.

with stage I-II SCC of the anus when adjustment is performed to account for baseline characteristics. However, on subgroup analysis, an OS benefit is noted for stage II patients but not for stage I patients. To our knowledge, this is the first SEER analysis evaluating this clinical question. The use of robust statistical techniques with propensity score matching and IPTW to reduce indication bias strengthen our findings. The addition or omission of chemotherapy in this patient population deserves careful scrutiny.

Chemotherapy is typically combined with RT based on two landmark trials comparing CRT and RT alone. A study from the EORTC randomized patients with T3$\mathrm{T} 4$ or node-positive disease to RT alone versus RT with concomitant fluorouracil and mitomycin C (5). The addition of chemotherapy improved complete remission rates $(80 \%$ vs. $54 \%, \mathrm{P}=0.02)$ and 5 -year locoregional control $(69 \%$ vs. $55 \%, \mathrm{P}=0.02)$ without a corresponding improvement in 5 -year OS (69\% vs. 64\%, $\mathrm{P}=0.17)$, though patients with $\mathrm{T} 1$ T2N0 disease were not included. A study from UKCCCR similarly found improved locoregional control with the addition of chemotherapy ( $61 \%$ vs. $39 \%, \mathrm{P}<0.001)$, though there was again no difference in 5 -year OS (65\% vs. 58\%, $\mathrm{P}=0.25$ ) (29). Of note, the rates of distant metastases on both studies were similar between the RT alone and CRT arms. It follows that chemotherapy principally improves locoregional events and likely optimizes outcomes by potentiating the local benefits of RT. Indeed, mitomycin $\mathrm{C}$ is a prodrug that undergoes bioreductive activation preferentially within hypoxic environments (30), resulting in synergism with RT, a hypothesis has been supported in in vitro studies (31). Accordingly, the hypoxic fraction within small anal cancers would be expected to be less than in larger cancers; therefore, there is a putative biological basis for treating smaller and less hypoxic anal cancers with RT alone.

In particular, we found that patients with T1N0 disease have no OS detriment when treated with RT alone. Previous retrospective series have had conflicting results with regards to optimal management of these patients. An unplanned subgroup analysis of 223 (38\%) patients on the UKCCCR trial did demonstrate that T1N0 patients $(\mathrm{P}=0.0352)$ and $\mathrm{T} 2 \mathrm{~N} 0$ patients $(\mathrm{P}=0.0049)$ benefitted from chemotherapy (7). In addition, with 
A

Kaplan-Meier Overall Survival: Stage I-II Anus SCC
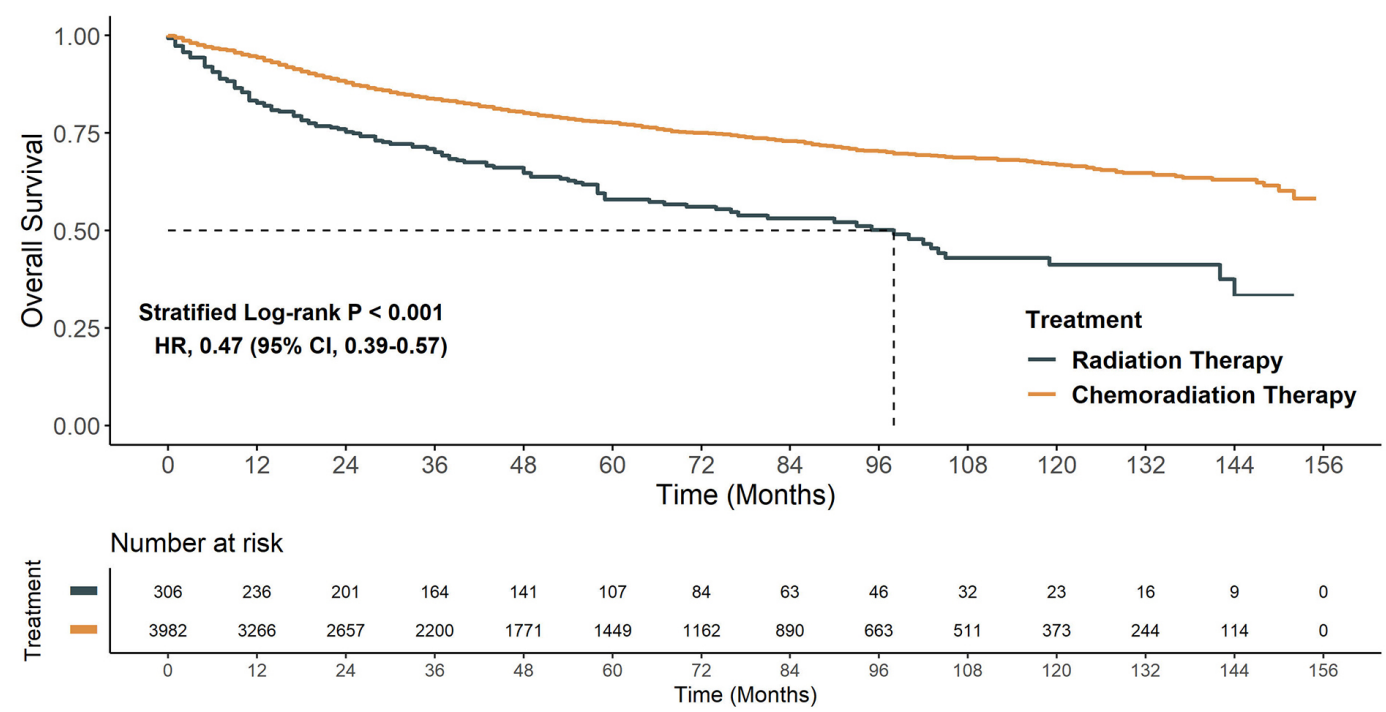

B

Kaplan-Meier Overall Survival: Stage I-II Anus SCC
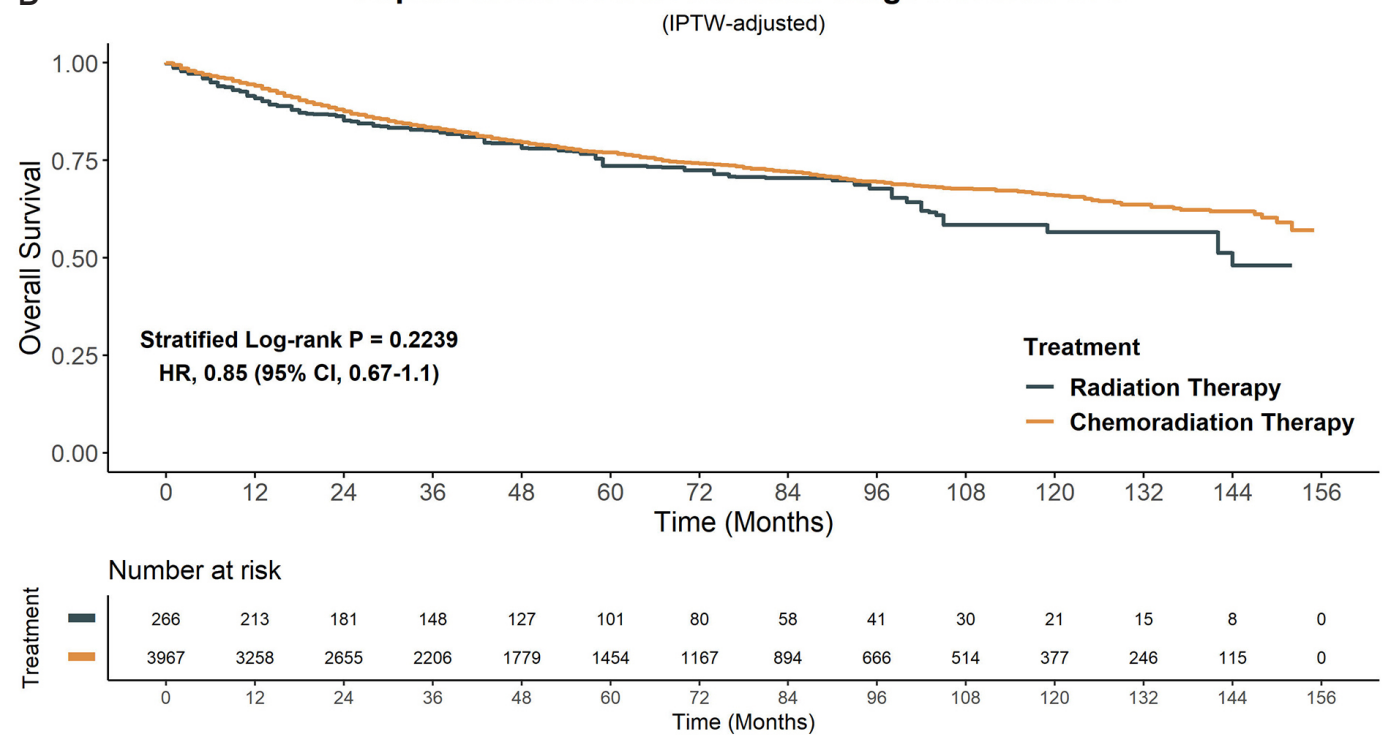

Figure 2 Overall survival curves for stage I-II SCC of the anus for the unadjusted groups (A) and IPTW-adjusted groups (B). SCC, squamous cell carcinoma; IPTW, inverse probability of treatment weighting.

13-year follow-up on the UKCCCR trial, there were 12.5 fewer anal cancer deaths for every 100 patients treated with chemoradiation. Moreover, the $9.1 \%$ increase in non-anal cancer deaths, which had been observed in the first 5 years of chemoradiation, had disappeared by 10 years (6). It is possible that chemoradiation demonstrates an oncological benefit even in T1N0 patients, but this did not lead to improved OS in our analysis due to non-anal cancer deaths, such as from cardiotoxicity or second malignancies. That said, the UKCCCR data suggests that this early toxicity detriment is overcome with improved late oncological control, so with longer follow-up an OS difference could emerge. On the other hand, Martenson and colleagues demonstrated a 5 -year $100 \%$ local control rate in T1-T2 patients receiving RT alone (13). In another series of 69 patients with Tis or $T 1$ anal canal carcinoma $\leq 1 \mathrm{~cm}$ treated with $\mathrm{RT}$ alone, the clinical complete response rate was $98 \%(65 / 66)$ and the 5 -year disease-free survival rate was 
A
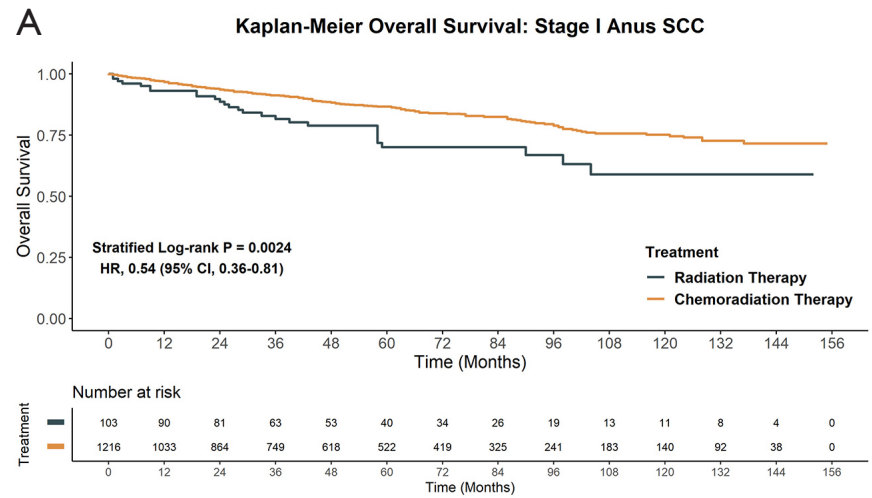

C

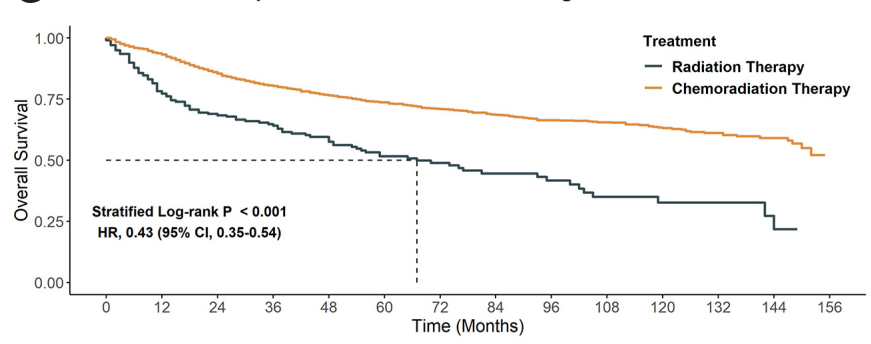

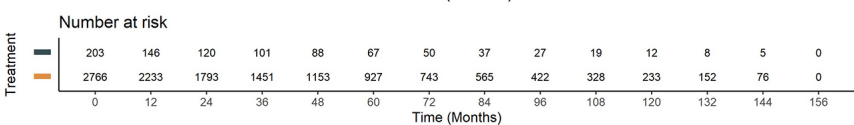
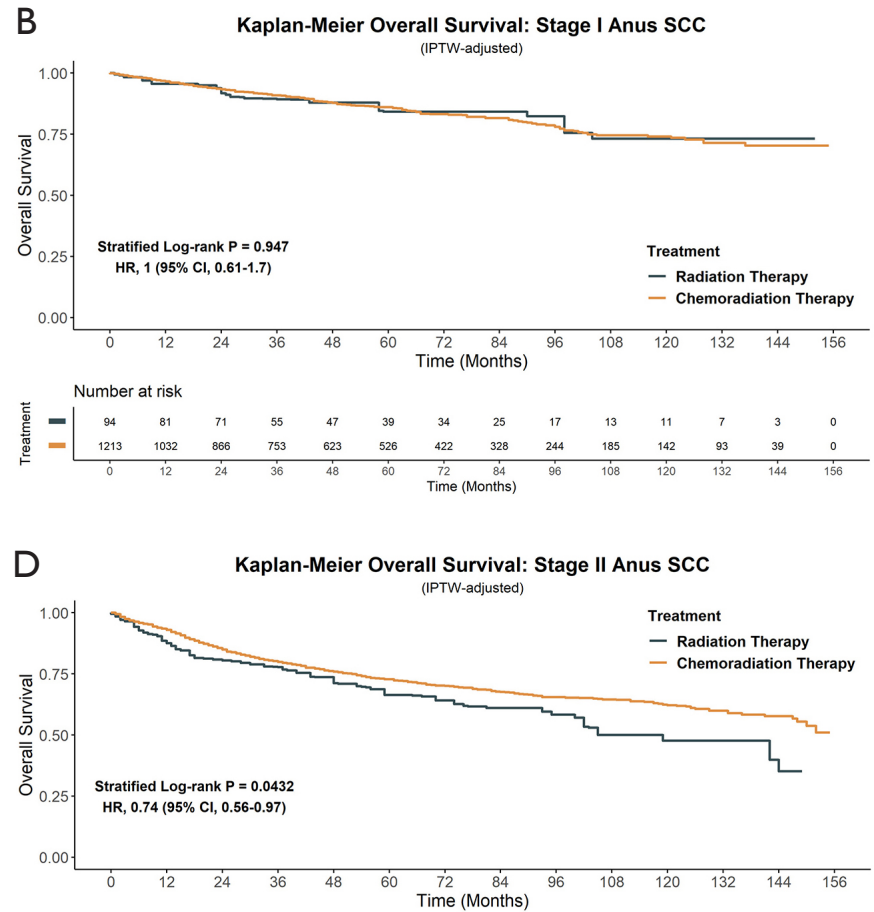

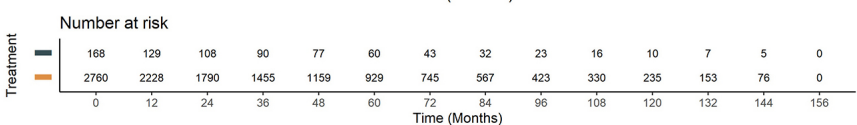

Figure 3 Overall survival curves for stage I SCC of the anus for the unadjusted groups (A) and IPTW-adjusted groups (B). Overall survival curves for stage II SCC of the anus for the unadjusted groups (C) and IPTW-adjusted groups (D). SCC, squamous cell carcinoma; IPTW, inverse probability of treatment weighting.

$89 \%$ (14). More recently, a SEER-Medicare study evaluated 200 patients age $\leq 65$ with stage I SCC anus who received CRT versus 99 with RT alone (16). Complementing the results reported herein, there was no difference in OS (HR $0.7,95 \%$ CI, 0.4-1.0) between the groups after propensity score adjustment. They also reported no differences in cause-specific survival, colostomy-free survival, and diseasefree survival, but did report higher risks of late bleeding [odds ratio (OR) 2.0, 95\% CI, 1.2-3.4] and proctitis (OR 2.1, 95\% CI, 1.2-3.5) in patients who underwent CRT. Interestingly, RT alone was much more commonly pursued in their analysis $(99 / 299,33 \%)$ than in our series $(103 / 1,319$, $8 \%$ ), suggesting that clinicians rarely consider RT alone in patients with T1N0 disease $\leq 65$ years of age but are more willing to do so in patients with advanced age and associated co-morbidities.

In contrast, we found that omission of chemotherapy in patients with stage II (T2-T3N0) disease leads to inferior OS. The need for multimodality therapy for T3N0 disease is not surprising, as it has previously been determined that primary tumor size $\geq 5 \mathrm{~cm}$ is a poor prognostic factor $(32,33)$. More intriguing is that patients with T2N0 disease $(>2 \mathrm{~cm}$ but $\leq 5 \mathrm{~cm}$ ) should not be treated with RT alone. This is corroborated by several previous studies. Zilli and colleagues examined 146 patients with predominantly T2N0 disease (80.1\%) treated with RT alone or CRT and demonstrated a crude improvement in cause-specific survival in patients undergoing CRT (94.9\%) compared to RT alone $(88.5 \%)(8)$. More recently, a report of the National Cancer Database (NCDB) identified 4,564 patients with T1-T2N0 patients with SCC of the anus (15). Amongst the $66.5 \%$ of patients with T2N0 disease, the 5 -year OS was $84.7 \%$ with CRT and $72.8 \%$ with RT alone $(\mathrm{P}<0.001)$. Similar to the present study, there was no difference in 5 -year OS amongst CRT (90.3\%) and RT (84.7\%) patients with T1N0 disease $(\mathrm{P}=0.11)$. They also found that the vast majority of patients (95.8\%) were treated with CRT, supporting the finding that clinicians are potentially over-treating patients for whom 
RT alone may be appropriate.

The current study, in combination with previous series, suggests that while CRT is likely necessary for T2N0 lesions, omission of chemotherapy may be appropriate in patients with T1N0 disease without a deleterious effect on OS. The NCCN guidelines do not offer RT alone as a suitable alternative to CRT, even in the most favorable of patients (17). Similarly, the most recent publication of the American College of Radiology (ACR) Appropriateness Criteria for Anal Cancer favor CRT despite encouraging results of RT alone (34). This is despite the fact that concurrent chemotherapy contributes significantly to treatment intolerance (18). As radiation techniques evolve and intensity-modulated radiation therapy (IMRT) becomes standard-of-care (35), there may be a corresponding role for de-intensification of systemic treatment. Based on our analysis, this may be most prudent in patients with very small $(<1.5 \mathrm{~cm})$, well-differentiated tumors.

Shortcomings of the present study include weaknesses inherent to retrospective analyses. In addition, the SEER registries lack detailed treatment information, limiting the prescription of both chemotherapy and radiation therapy (CRT) to binary variables. It is possible that patients undergoing RT may have received dose-escalation to account for the lack of systemic therapy. Information on human immunodeficiency virus (HIV) status and human papillomavirus (HPV) were not available. In addition, medical co-morbidities precluding the prescription of chemotherapy were not available from the database and could not be accounted for in our statistical model. Moreover, local control is an important endpoint in the treatment of anal cancer, as salvage for treatment failure is an abdominopelvic resection (APR), which can have significant negative detriments in terms of patient quality of life. Unfortunately, this endpoint is not provided in the SEER registries. Indeed, the SEER registries only provide OS as a clinical endpoint. The SEER registries are quality-controlled but the data are reliant on accurate coding and reporting, which may be prone to bias. Finally, positron emission tomography/computed tomography (PET/CT) has recently become more widespread in the staging of patients with anal cancer, and studies suggest that when compared to conventional imaging, the use of PET may result in a change of nodal staging in up to $28 \%$ of patients (36). It is possible that some patients included in the present analysis did not undergo PET/CT and were thus understaged. On the other hand, the large number of patients on the SEER registry allows for robust analysis with sufficient events to perform MVA. As SEER accounts for $34.6 \%$ of the population, it can be considered representative of the entire population.

To conclude, we present a population-based study demonstrating an OS benefit in patients with stage II SCC of the anus treated with CRT compared to RT alone, with no such benefit in patients with stage I disease. Our findings are hypothesis-generating, and prospective evidence is needed to optimize clinical decision-making. In patients with stage I anus cancer, clinicians should weigh the potential improvement in clinical outcomes with the added toxicity of systemic therapy. Definitive RT as monotherapy may be appropriate in patients with tumors less than $2 \mathrm{~cm}$.

\section{Acknowledgments}

Funding: None.

\section{Footnote}

Reporting Checklist: The authors have completed the STROBE reporting checklist. Available at http://dx.doi. org/10.21037/jgo-20-530

Peer Review File: Available at http://dx.doi.org/10.21037/ jgo-20-530

Conflicts of Interest: All authors have completed the ICMJE uniform disclosure form (available at: http://dx.doi. org/10.21037/jgo-20-530). The authors have no conflicts of interest to declare.

Ethical Statement: The authors are accountable for all aspects of the work in ensuring that questions related to the accuracy or integrity of any part of the work are appropriately investigated and resolved. This study was exempt from institutional review board approval because the data set was de-identified and publicly available. The study was conducted in accordance with the Declaration of Helsinki (as revised in 2013).

Open Access Statement: This is an Open Access article distributed in accordance with the Creative Commons Attribution-NonCommercial-NoDerivs 4.0 International License (CC BY-NC-ND 4.0), which permits the noncommercial replication and distribution of the article with the strict proviso that no changes or edits are made and the original work is properly cited (including links to both the 
formal publication through the relevant DOI and the license). See: https://creativecommons.org/licenses/by-nc-nd/4.0/.

\section{References}

1. Siegel RL, Miller KD, Jemal A. Cancer statistics, 2020. CA Cancer J Clin 2020;70:7-30.

2. Nelson RA, Levine AM, Bernstein L, et al. Changing patterns of anal canal carcinoma in the United States. J Clin Oncol 2013;31:1569-75.

3. Gordon PH. Squamous-cell carcinoma of the anal canal. Surg Clin North Am 1988;68:1391-9.

4. Nigro ND, Seydel HG, Considine B, et al. Combined preoperative radiation and chemotherapy for squamous cell carcinoma of the anal canal. Cancer 1983;51:1826-9.

5. Bartelink H, Roelofsen F, Eschwege F, et al. Concomitant radiotherapy and chemotherapy is superior to radiotherapy alone in the treatment of locally advanced anal cancer: results of a phase III randomized trial of the European Organization for Research and Treatment of Cancer Radiotherapy and Gastrointestinal Cooperative Groups. J Clin Oncol 1997;15:2040-9.

6. Northover J, Glynne-Jones R, Sebag-Montefiore D, et al. Chemoradiation for the treatment of epidermoid anal cancer: 13-year follow-up of the first randomised UKCCCR Anal Cancer Trial (ACT I). Br J Cancer 2010;102:1123-8.

7. Bosset JF, Pavy JJ, Roelofsen F, et al. Combined radiotherapy and chemotherapy for anal cancer. EORTC Radiotherapy and Gastrointestinal Cooperative Groups. Lancet 1997;349:205-6.

8. Zilli T, Schick U, Ozsahin M, et al. Node-negative T1T2 anal cancer: radiotherapy alone or concomitant chemoradiotherapy? Radiother Oncol 2012;102:62-7.

9. De Bari B, Lestrade L, Pommier P, et al. Could concomitant radio-chemotherapy improve the outcomes of early-stage node negative anal canal cancer patients? A retrospective analysis of 122 patients. Cancer Invest 2015;33:114-20.

10. Deniaud-Alexandre E, Touboul E, Tiret E, et al. Results of definitive irradiation in a series of 305 epidermoid carcinomas of the anal canal. Int J Radiat Oncol Biol Phys 2003;56:1259-73.

11. Myerson RJ, Kong F, Birnbaum EH, et al. Radiation therapy for epidermoid carcinoma of the anal canal, clinical and treatment factors associated with outcome Radiother Oncol 2001;61:15-22.

12. Allal A, Kurtz JM, Pipard G, et al. Chemoradiotherapy versus radiotherapy alone for anal cancer: a retrospective comparison. Int J Radiat Oncol Biol Phys 1993;27:59-66.

13. Martenson JA Jr, Gunderson LL. External radiation therapy without chemotherapy in the management of anal cancer. Cancer 1993;71:1736-40.

14. Ortholan C, Ramaioli A, Peiffert D, et al. Anal canal carcinoma: early-stage tumors $<$ or $=10 \mathrm{~mm}$ ( $\mathrm{T} 1$ or Tis): therapeutic options and original pattern of local failure after radiotherapy. Int J Radiat Oncol Biol Phys 2005;62:479-85.

15. Youssef I, Osborn V, Lee A, et al. Survival benefits and predictors of use of chemoradiation compared with radiation alone for early stage (T1-T2N0) anal squamous cell carcinoma. J Gastrointest Oncol 2019;10:616-22.

16. Buckstein M, Arens Y, Wisnivesky J, et al. A PopulationBased Cohort Analysis of Chemoradiation Versus Radiation Alone for Definitive Treatment of Stage I Anal Cancer in Older Patients. Dis Colon Rectum 2018;61:787-94.

17. Network NCC. Anal Carcinoma (Version 2.2020). Available online: https://www.nccn.org/professionals/ physician_gls/PDF/anal.pdf

18. Ajani JA, Winter KA, Gunderson LL, et al. Fluorouracil, mitomycin, and radiotherapy vs. fluorouracil, cisplatin, and radiotherapy for carcinoma of the anal canal: a randomized controlled trial. JAMA 2008;299:1914-21.

19. Austin PC. Goodness-of-fit diagnostics for the propensity score model when estimating treatment effects using covariate adjustment with the propensity score. Pharmacoepidemiol Drug Saf 2008;17:1202-17.

20. Efron B. Logistic regression, survival analysis, and the Kaplan-Meier curve. J Am Stat Assoc 1988;83:414-25.

21. Almahariq MF, Quinn TJ, Siddiqui ZA, et al. Postmastectomy radiotherapy is associated with improved overall survival in T3N0 patients who do not receive chemotherapy. Radiother Oncol 2020;145:229-37.

22. Mankuzhy NP, Almahariq MF, Siddiqui ZA, et al. The Role of Postoperative Radiation Therapy for pN2 Nonsmall-cell Lung Cancer. Clin Lung Cancer 2021;22:e5-e17.

23. Van Buuren S, Brand JPL, Groothuis-Oudshoorn CGM, et al. Fully conditional specification in multivariate imputation. J Stat Comput Simul 2006;76:1049-64.

24. Rosenbaum PR. Propensity score. Encyclopedia of Biostatistics. Chichester, UK: Wiley, 2005.

25. Cole SR, Hernan MA. Constructing inverse probability weights for marginal structural models. Am J Epidemiol 2008;168:656-64. 
26. Austin PC, Stuart EA. Moving towards best practice when using inverse probability of treatment weighting (IPTW) using the propensity score to estimate causal treatment effects in observational studies. Stat Med 2015;34:3661-79.

27. Thompson AB, Quinn TJ, Siddiqui ZA, et al. Addition of radiotherapy to surgery and chemotherapy improves survival in localized malignant pleural mesothelioma: A Surveillance, Epidemiology, and End Results (SEER) study. Lung Cancer 2020;146:120-6.

28. Quinn TJ, Almahariq MF, Siddiqui ZA, et al. Trimodality therapy for atypical teratoid/rhabdoid tumor is associated with improved overall survival: A surveillance, epidemiology, and end results analysis. Pediatr Blood Cancer 2019;66:e27969.

29. Epidermoid anal cancer: results from the UKCCCR randomised trial of radiotherapy alone versus radiotherapy, 5-fluorouracil, and mitomycin. UKCCCR Anal Cancer Trial Working Party. UK Co-ordinating Committee on Cancer Research. Lancet 1996;348:1049-54.

30. Mistry IN, Thomas M, Calder EDD, et al. Clinical Advances of Hypoxia-Activated Prodrugs in Combination With Radiation Therapy. Int J Radiat Oncol Biol Phys 2017;98:1183-96.

31. Dobrowsky W, Dobrowsky E, Rauth AM. Mode of

Cite this article as: Parzen JS, Vayntraub A, Squires B, Almahariq MF, Thompson AB, Robertson JM, Kabolizadeh P, Quinn TJ. A population-based analysis of chemoradiation versus radiation alone in the definitive treatment of patients with stage I-II squamous cell carcinoma of the anus. J Gastrointest Oncol 2021;12(2):831-844. doi: 10.21037/jgo-20-530 interaction of 5-fluorouracil, radiation, and mitomycin C: in vitro studies. Int J Radiat Oncol Biol Phys 1992;22:875-80.

32. Kapacee ZA, Susnerwala S, Wise M, et al. Chemoradiotherapy for squamous cell anal carcinoma: a review of prognostic factors. Colorectal Dis 2016;18:1080-6.

33. Ajani JA, Winter KA, Gunderson LL, et al. Prognostic factors derived from a prospective database dictate clinical biology of anal cancer: the intergroup trial (RTOG 98-11). Cancer 2010;116:4007-13.

34. Expert Panel on Radiation Oncology-Rectal/Anal C, Hong TS, Pretz JL, et al. ACR Appropriateness Criteria(R)-Anal Cancer. Gastrointest Cancer Res 2014;7:4-14.

35. Kachnic LA, Winter K, Myerson RJ, et al. RTOG 0529: a phase 2 evaluation of dose-painted intensity modulated radiation therapy in combination with 5 -fluorouracil and mitomycin-C for the reduction of acute morbidity in carcinoma of the anal canal. Int J Radiat Oncol Biol Phys 2013;86:27-33.

36. Jones M, Hruby G, Solomon M, et al. The Role of FDGPET in the Initial Staging and Response Assessment of Anal Cancer: A Systematic Review and Meta-analysis. Ann Surg Oncol 2015;22:3574-81. 


\section{Supplementary}
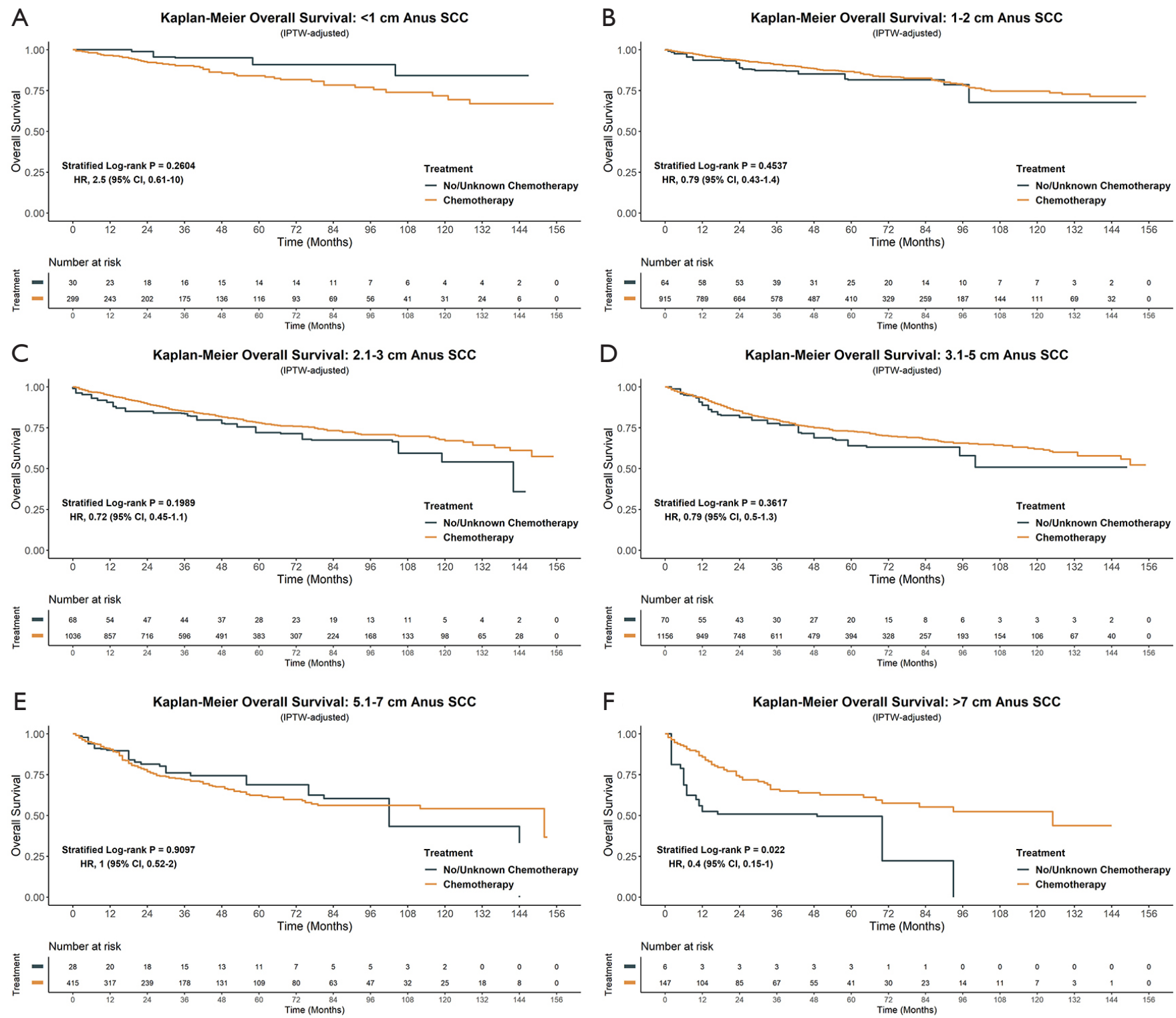

Figure S1 Overall survival curves for SCC of the anus for IPTW-adjusted groups with tumors $<1 \mathrm{~cm}(\mathrm{~A}), 1-2 \mathrm{~cm}(\mathrm{~B}), 2.1-3 \mathrm{~cm}(\mathrm{C}), 3.1-$ $5 \mathrm{~cm}(\mathrm{D}), 5.1-7 \mathrm{~cm}(\mathrm{E})$, and $>7 \mathrm{~cm}(\mathrm{~F})$. 